\title{
Alcohol, drogas, sexo y violencia: riesgos y consecuencias para la salud entre los jóvenes turistas británicos en las Islas Baleares
}

\section{Alcohol, drugs, sex and violence: health risks and consequences in young British holidaymakers to the Balearics}

Karen Hughes ${ }^{1}$; Mark A Bellis ${ }^{1}$; Gayle Whelan ${ }^{1}$; Amador Calafat ${ }^{2}$; Montse Juan²; Nicole Blay²
${ }^{1}$ Centre for Public Health, Liverpool John Moores University. UK 2 Irefrea (Instituto Europeo de Estudios en Prevención). España

Enviar correspondencia a:

Karen Hugues.

Centre for Public Health, Liverpool John Moores University, 5th

Floor Kingsway House, Hatton Garden, Liverpool, L3 2AJ, UK

Tel: +44 (0)151 231 8723, Email: k.e.hughes@ljmu.ac.uk

\section{RESUMEN}

Antecedentes: Consumo de sustancias, conducta sexual de riesgo y violencia son los principales problemas de salud que sufren los jóvenes en la actualidad. Todo ello supone una gran labor preventiva en el Reino Unido, pero escasea la información sobre la prevención en los centros turisticos internacionales. Métodos:

Realizamos un estudio transversal entre 1033 turistas británicos, de edades entre los 16 y los 35 años en los aeropuertos de Ibiza y Mallorca (España). El estudio analizó la frecuencia de consumo de alcohol, drogas, borracheras, actividad sexual y experiencias negativas en Inglaterra y en el extranjero durante las vacaciones. Resultados: El consumo de drogas ilegales fue más frecuente en lbiza. Más de la mitad $(53,9 \%)$ admitieron haber consumido al menos una droga ilegal en vacaciones (frente al 13,9\% en Mallorca) y un tercio consumieron más de una. Sin embargo, los turistas de Mallorca se embriagaron con más frecuencia y con peores consecuencias (discusiones, peleas, daños involuntarios, enfermedades y necesidad de recibir asistencia médica). En ambos lugares la frecuencia del consumo de sustancias aumentó en vacaciones. La cuarta parte de los que viajaron sin pareja mantuvieron relaciones sexuales en vacaciones, y de ellos un tercio no utilizó siempre condones. La embriaguez frecuente se asoció a peleas, daños involuntarios y relaciones sexuales con una nueva pareja. El consumo de drogas ilegales, particularmente el policonsumo de drogas, se asoció a peleas y sexo sin protección. Conclusiones: Los turistas jóvenes afrontan considerables riesgos de salud en vacaciones. Identificar los centros turísticos asociados con las diferentes conductas de riesgo es fundamental para realizar campañas de protección de la salud y ejecutar medidas de reducción del daño.

Palabras clave: alcohol, drogas recreativas, violencia, conducta de riesgo, viajes, vida nocturna.

\section{ABSTRACT}

Background: Substance use, risky sexual behaviour and violence are among the key youth health issues today. Whilst they are the focus of much prevention work in the UK, relatively little information is available to inform prevention in international holiday resorts, where young people can take the greatest risks with their health.

Methods: We conducted a cross-sectional survey of 1033 British holidaymakers aged 16-35 in Ibiza and Majorca airports (Spain). Surveys covered frequency of alcohol and drug use at home and abroad, and drunkenness, sexual activity and experience of a range of negative experiences on holiday. Results: Illicit drug use was more common in Ibiza. Over half (53.9\%) of visitors to Ibiza reported using at least one drug on holiday (compared with 13.9\% in Majorca) and over a third reported using more than one drug. However visitors to Majorca reported more frequent drunkenness and more negative outcomes (arguments, fighting, unintentional injury, illness and requiring medical attention). In both locations, frequency of substance use increased on holiday. A quarter of those travelling without a partner reported having had sex during their holiday, and of these a third did not consistently use condoms. Frequent drunkenness was associated with fighting, unintentional injury and sex with a new partner. Illicit drug use, particularly polydrug use, was associated with fighting and unprotected sex. Conclusions: Young holidaymakers face significant health risks on holiday. Understanding which resorts are associated with which types of health risk behaviour is critical in targeting health promotion and harm reduction measures appropriately.

Key words: alcohol, recreational drugs, violence, risk behaviour, travel, nightlife. 


\section{INTRODUCCIÓN}

E abuso del alcohol, el consumo de drogas ilegales, la conducta sexual de riesgo y la violencia son algunos de los mayores peligros que hoy acosan la salud de los jóvenes. En particular, entre los jóvenes británicos, los niveles de consumo de sustancias, conducta sexual de riesgo asociada a los mismos y agresión exceden los de muchos países europeos, ya desde la adolescencia ${ }^{1,2}$. Este tipo de conducta continúa a menudo durante la primera edad adulta. El consumo compulsivo de alcohol está muy extendido en la vida nocturna británica ${ }^{3}$, y uno de cada cinco jóvenes entre los 16 y los 24 años ha consumido drogas en el último año $0^{4}$. En este grupo de edad se registran más de un millón de incidentes violentos al año ${ }^{5}$, y hubo 150.000 nuevos casos de infecciones de transmisión sexual (ITS) en $2007^{6}$. Tanto la violencia ${ }^{7}$ como la conducta sexual de riesgo tienen mucho que ver con el consumo de sustancias. Por ejemplo, casi la mitad de la violencia registrada en Inglaterra y Gales se comete bajo los efectos del alcohol ${ }^{5}$, y tanto el alcohol como las drogas ilegales se utilizan de forma consciente para facilitar las relaciones sexuales y aumentar el placer ${ }^{8}$.

La creciente carga del consumo de sustancias, conducta sexual de riesgo y violencia en Gran Bretaña ha colocado estos temas entre los más importantes de las agendas gubernamentales, dando lugar a programas de gran alcance para reducir los daños provocados por el alcohol ${ }^{9}$ y el consumo de drogas ${ }^{10}$, evitar la violencia ${ }^{11}$ y promover la salud sexual ${ }^{12}$. Sin embargo, estos programas se centran sobre todo en proteger a los jóvenes en Gran Bretaña. Apenas se ha dedicado atención a la conducta y salud de los jóvenes cuando viajan al extranjero. Y ello a pesar de que los jóvenes casi siempre asumen los riesgos de salud más graves en vacaciones. Las investigaciones demuestran que el consumo de sustancias y la actividad sexual entre los jóvenes aumentan durante los períodos vacacionales ${ }^{13-16}$. Los centros turísticos internacionales dirigidos a los jóvenes giran casi siempre en torno a la vida nocturna, con elevadas concentraciones de bares y clubes que ofrecen la oportunidad de relaciones hedonistas todas las noches de la semana ${ }^{17}$. Entre los turistas jóvenes disminuyen las restricciones sociales y aumentan las ocasiones de conocer gente y experimentar ideas nuevas ${ }^{18}$. Los jóvenes aprecian mucho estos períodos, pero los excesos vacacionales perjudican la salud (p.ej., intoxicación por drogas $^{19}$ ), sobrecargan los servicios de salud y de orden público de los centros turísticos ${ }^{19-22}$ y los de los países de origen de los turistas cuando las consecuencias de las conductas de riesgo (p.ej., ITS ${ }^{23}$ ) regresan con el viajero. Por tanto, el conocimiento de las conductas de riesgo entre los turistas es fundamental para desarrollar medidas apropiadas de protección de la salud y de reducción de daños.

Gracias al progresivo abaratamiento y la facilidad de los viajes internacionales en las últimas décadas ${ }^{24}$, los jóvenes pasan cada vez más tiempo en el extranjero. En el año 2006 los jóvenes británicos entre 16 y 34 años realizaron casi 12 millones de viajes al extranjero ${ }^{25}$. España es el destino más popular entre los jóvenes viajeros británicos; en especial las islas Baleares de Mallorca e Ibiza, que recibieron a casi tres

\section{INTRODUCTION}

A Icohol misuse, illicit drug use, risky sexual behaviour and violence pose some of the biggest challenges to young people's health today. In particular in young British populations, levels of substance use and related risky sexual behaviour and aggression exceed those in many other European countries, even during teenage years. ${ }^{1,2}$ Such behaviours can continue long into young adulthood. Thus binge drinking in British nightlife is widespread, ${ }^{3}$ whilst one in five 16-24 year olds have used drugs in the last year. ${ }^{4}$ This age group also commits over a million incidents of violence each year, ${ }^{5}$ and accounted for around 150,000 new diagnoses of sexually transmitted infections (STIs) in $2007 .{ }^{6}$ Both violence ${ }^{7}$ and risky sexual behaviour are strongly associated with substance use. For example, almost half of all violence in England and Wales is committed under the influence of alcohol, ${ }^{5}$ and both alcohol and illicit drugs are often used specifically to facilitate sexual encounters and enhance sexual pleasure. ${ }^{8}$

The growing burden of substance use, risky sexual behaviour and violence in the UK has raised such issues to the top of government agendas, with major programmes underway to reduce the harms caused by drinking ${ }^{9}$ and drug use, ${ }^{10}$ prevent violence ${ }^{11}$ and promote sexual health. ${ }^{12}$ However, these programmes focus largely on engaging with and protecting young people in the UK. Little attention has targeted the behaviour and health of young people when they travel abroad. Despite this, young people often take the greatest risks with their health on holiday. Research has shown that substance use and sexual activity in young people increases during holiday periods..$^{13-16}$ International resorts that target young people are often oriented around nightlife, with high concentrations of bars and nightclubs offering opportunities for hedonistic partying every night of the week. ${ }^{17}$ For young holidaymakers, social restraints can be low and opportunities for meeting new people and experimenting with new ideas increased..$^{18}$ Whilst these periods are valued by young people, excessive holiday behaviours can damage health (e.g. drug poisoning ${ }^{19}$ ), and place large burdens on health and criminal justice agencies in resorts, ${ }^{19-22}$ and those in tourists' home countries as the consequences of holiday behaviours (e.g. $\mathrm{STIs}^{23}$ ) return home with the traveller. Thus understanding holidaymakers' health risk behaviours is critical in developing appropriate health promotion and harm reduction measures.

With international travel having become cheaper and easier over the last few decades, ${ }^{24}$ young people are spending more time abroad. In 2006, 16-34 year olds from the UK made almost 12 million trips abroad. ${ }^{25}$ Spain is the most popular overseas destination for young British travellers; particularly the Balearic Islands of Majorca and Ibiza that received around three million visits by British nationals in $2007 .{ }^{26}$ To explore the health risk behaviours 
millones de británicos en el $2007^{26}$. Para analizar las conductas de riesgo de los turistas británicos en estos destinos, realizamos un estudio entre 1033 jóvenes de edades comprendidas entre 18 y 35 años que pasaron sus vacaciones en Mallorca e lbiza en el verano del 2007.

\section{MÉTODOS}

Se confeccionó un cuestionario breve y anónimo para estudiar: la frecuencia de consumo de alcohol, tabaco y drogas (cannabis, éxtasis, cocaína, anfetamina, ketamina y GHB [gamahidroxibutirato]) en el pais de origen y durante las vacaciones; la conducta sexual en vacaciones; y las experiencias negativas, como enfermedades, discusiones, agresiones físicas, daño no intencionado, necesidad de asistencia médica y robo de objetos. El cuestionario se elaboró partiendo de herramientas validadas de antemano para estudiar el comportamiento en vacaciones ${ }^{14,27}$. Para medir los niveles de consumo de alcohol, se preguntó a los participantes cuántos dias de vacaciones habian estado borrachos y, en consecuencia, la embriaguez fue definida por ellos. En el caso del alcohol y las drogas, el consumo habitual en Gran Bretaña se refirió a los últimos 12 meses. El sexo sin protección se definió como el mantenimiento de relaciones sexuales sin preservativo. La conducta sexual de riesgo se refiere en este estudio a la práctica del sexo sin protección o con múltiples parejas durante las vacaciones.

Se contrataron y entrenaron encuestadores británicos experimentados para realizar la encuesta, que se realizó en las zonas de salida de los aeropuertos de Mallorca (del 30 de julio a 5 de agosto de 2007) e lbiza (del 21 al 28 de agosto de 2007). Ambos períodos correspondían al pico de la temporada vacacional en Europa, una vez terminados los cursos de educación media y superior en Gran Bretaña. Se indicó a los encuestadores que se dirigiesen a todos los individuos de edades comprendidas entre los 16 y los 35 años, que viajasen sin niños ni familiares mayores y que fuesen a coger vuelos de regreso a Gran Bretaña. En cada lugar se estableció una muestra de observación de 500 participantes, sobre la base de estudios previos ${ }^{14,27}$. La encuesta formó parte de un estudio más extenso que incluia turistas alemanes y españoles en ambas ubicaciones. En todas las nacionalidades se registraron casos de consumo de sustancias y violencia ${ }^{28,29}$. Este artículo ofrece análisis adicionales sobre los turistas británicos que, en estudios anteriores, presentaron niveles más altos de consumo de sustancias y de violencia que los de otras nacionalidades ${ }^{28,29}$.

En conjunto se abordó a 1399 personas y se les preguntó si tenían tiempo para rellenar un breve cuestionario (Mallorca $n=649$, Ibiza $n=750)$. De ellos, el 15,7\% $(n=102)$ en Mallorca y $21,2 \%(n=159)$ en lbiza se negaron antes de que se les explicase en qué consistía el cuestionario. A los que dijeron que tenían tiempo, se les explicó el cuestionario, se les garantizó el anonimato y la confidencialidad y se les pidió que participasen. Se mostraron dispuestos a participar el $98,2 \%(n=537)$ en Mallorca y el $92,6 \%(n=547)$ en Ibiza. A los participantes se les entregó un cuestionario, una of British tourists to these destinations, we surveyed 1033 18-35 year olds holidaying in Majorca and Ibiza in summer 2007.

\section{METHODS}

A short, anonymous questionnaire was designed to explore: frequency of alcohol, tobacco and drug use (cannabis, ecstasy, cocaine, amphetamine, ketamine and GHB [gammahydroxybutyrate]) at home and during the holiday; sexual behaviour on holiday; and negative holiday experiences including illness, arguments, physical fighting, unintentional injury, need for medical attention, and theft of belongings. The questionnaire was developed from prevalidated tools for surveying holiday behaviour. ${ }^{14,27}$ To gain a measure of levels of alcohol use, participants were asked to identify on how many days of their holiday they considered themselves to have been drunk, and consequently drunkenness was self-defined. For alcohol and drugs, current use in the UK was defined as having used in the last 12 months. Unprotected sex is defined as having sex without a condom. Risky sexual behaviour here refers to having unprotected sex or multiple sexual partners on holiday.

Experienced researchers from the UK were recruited and trained to carry out the survey, which took place in the departure areas of Majorca (30th July to 5th August 2007) and Ibiza (21st to 28th August 2007) airports. Both periods fell within the peak European holiday season and outside of regular school and higher education terms in most of the UK. Researchers were instructed to target all individuals who appeared to be aged 16 to 35 and travelling without children or older relatives, who were waiting to check in to flights bound for the UK. Based on previous studies, ${ }^{14,27}$ a target sample of 500 participants was set for each location. The survey formed part of a wider study that also included German and Spanish holidaymakers to each location. Findings on substance use and violence across all nationalities are reported elsewhere. ${ }^{28,29}$ This paper presents additional analyses of British holidaymakers who, in earlier analyses, displayed higher levels of both substance use and violence than other nationalities. ${ }^{28,29}$

Overall, 1399 individuals were approached and asked if they had time to complete a short questionnaire (Majorca $n=649$, Ibiza $n=750)$. Of these, $15.7 \%(n=102)$ in Majorca and $21.2 \%(n=159)$ in lbiza declined before the nature of the survey was explained to them. Those stating they had time were provided with an explanation of the survey, assured of its anonymity and confidentiality and asked if they would be willing to participate. Consent was given by 98.2\% $(n=537)$ in Majorca and 92.6\% $(n=547)$ in Ibiza. Participants were handed a questionnaire, clip-board, pen and envelope and asked to self-complete the questionnaire 
tablilla, un boligrafo y un sobre y se les pidió que rellenasen el cuestionario y lo introdujesen en el sobre, que recogerían los encuestadores. Los encuestadores debían permanecer a discreta distancia para que los participantes cubriesen el cuestionario en privado, pero contasen con ayuda en caso necesario.

Los cuestionarios cubiertos se enviaron a Gran Bretaña y se introdujeron en una base de datos utilizando el programa SPSS v14. Se excluyeron 51 cuestionarios porque los participantes no entraban en el rango de edades, estaban incompletos, los datos eran incoherentes o estaban llenos de borrones. Y así, la muestra final fue de 1033 (Mallorca: $n=505$; Ibiza $n=528$ ). Las diferencias entre las muestras se analizaron utilizando la distribución ji cuadrado, que se usó también para analizar las diferencias en las conductas vacacionales de riesgo entre los lugares de estudio y entre géneros, y los cambios en la frecuencia del consumo de sustancias en vacaciones en comparación con el del resto del año. Sin embargo, para identificar factores de predicción de participación en altercados, daños no intencionados, relaciones sexuales y conducta sexual de riesgo, se utilizó la regresión logística condicional para prevenir factores de confusión.

\section{RESULTADOS}

Los participantes encuestados en Mallorca eran más jóvenes y sus vacaciones fueron más largas que los de lbiza (Tabla 1). Sin embargo, en ambos casos la vida nocturna fue la razón más común para elegir el destino vacacional (Mallorca 82,9\%; Ibiza 89,0\%, $p<0,01$ ). La gran mayoría de visitantes de ambos lugares consumían alcohol habitualmente (consumo en Gran Bretaña en los doce meses anteriores), aunque en el caso de otras sustancias el consumo era mucho más alto en la muestra de lbiza. Por ejemplo, el 33,5\% de visitantes de lbiza eran consumidores habituales de éxtasis y el $33,7 \%$ de cocaina, frente al 7,3\% y al 9,3\% de los visitantes de Mallorca, respectivamente $(p<0.001)$.

Los niveles de consumo de drogas durante las vacaciones también fueron más altos en Ibiza (Tabla 2), donde el $44,2 \%$ admitió haber consumido éxtasis durante su estancia y el $34,2 \%$ cocaina, frente al $4,0 \%$ y el $7,5 \%$ respectivamente en Mallorca $(p<0.001)$. Más de la mitad $(53,9 \%)$ de los visitantes de lbiza consumieron al menos una droga ilegal durante las vacaciones (frente al 13,9\% en Mallorca) y un tercio consumió más de una droga. Sin embargo, los visitantes de Mallorca se embriagaron más frecuentemente en vacaciones: el 60,8\% se embriagó cinco o más veces por semana en vacaciones, frente al $53,2 \%(p<0.01)$. Aproximadamente nueve de cada diez visitantes de ambos lugares se embriagaron al menos una vez en vacaciones. La embriaguez frecuente y el consumo de cannabis y cocaína fueron más frecuentes entre los hombres en ambos lugares, igual que el consumo de éxtasis en lbiza.

Las experiencias negativas en vacaciones como enfermedades, discusiones, agresiones físicas, daños no intencionados y necesidad de acudir al médico/o al hospital fueron más frecuentes en la muestra de Mallorca. Por ejemplo, el 11,6\% and seal it in the envelope for collection by researchers. Researchers were instructed to stand a discrete distance away to permit the questionnaire to be completed in private yet allow respondents to seek assistance if required.

Completed questionnaires were returned to the UK and entered into a database using SPSS v14. At this point, 51 questionnaires were excluded due to participants being outside the target age range, or for questionnaires being incomplete, inconsistent or defaced. Thus, the final sample was 1033 (Majorca: $n=505$; Ibiza $n=528$ ). Differences between samples were analysed utilising chi squared. Chi squared was also used to explore differences in holiday health risk behaviours between study locations and genders, and changes in frequency of substance use on holiday compared with use at home. However, to identify predictive factors for involvement in physical fighting, unintentional injury, having sex and risky sexual behaviour, backward conditional logistic regression was used to control for confounding factors.

\section{RESULTS}

Participants surveyed in Majorca were younger and reported a longer length of stay than those surveyed in Ibiza (Table 1). However, for both samples, nightlife was the most common reason selected for choosing the holiday destination (Majorca 82.9\%, Ibiza 89.0\%, p<0.01). The vast majority of visitors to both locations were current alcohol consumers (i.e. reported use in the UK in the last twelve months), yet for most other substances current use was significantly higher among the Ibiza sample. For example, $33.5 \%$ of visitors to lbiza reported being current ecstasy users and $33.7 \%$ current cocaine users, compared with $7.3 \%$ and $9.3 \%$ of visitors to Majorca respectively $(p<0.001)$.

Levels of drug use during the holiday were also higher in Ibiza (Table 2). Here $44.2 \%$ reported using ecstasy during their stay and $34.2 \%$ cocaine, compared with $4.0 \%$ and $7.5 \%$ respectively in Majorca $(p<0.001)$. Over half $(53.9 \%)$ of all visitors to lbiza reported using at least one illicit drug during their holiday (compared with 13.9\% in Majorca) and over a third reported using more than one drug. However, visitors to Majorca reported more frequent drunkenness during the holiday; $60.8 \%$ reported being drunk five or more times per week on holiday compared with $53.2 \%$ in Ibiza $(p<0.01)$. Around nine in ten visitors to both locations reported getting drunk at least once during their holiday. Frequent drunkenness and use of cannabis and cocaine were more commonly reported by males in both locations, as was ecstasy use in Ibiza.

Negative holiday experiences including illness, arguments, physical fighting, unintentional injury, 
Tabla 1: Caracteristicas de la muestra según lugar de estudio

Table 1: Sample characteristics by location of survey

\begin{tabular}{|c|c|c|c|}
\hline & \multicolumn{2}{|c|}{ Lugar de estudio / Location of survey } & \multirow[b]{2}{*}{$P$} \\
\hline & Mallorca / Majorca & Ibiza & \\
\hline Muestra (n) / Sample (n) & 505 & 528 & \\
\hline \multicolumn{4}{|l|}{ Género (\%) / Gender (\%) } \\
\hline Hombre / Male & 48.7 & 45.1 & \\
\hline Mujer / Female & 51.3 & 54.9 & NS \\
\hline \multicolumn{4}{|l|}{ Grupo de edad (\%)'/ Age group } \\
\hline r & 61.6 & 12.1 & \\
\hline $20-25$ & 33.9 & 56.4 & \\
\hline $26-35$ & 4.6 & 31.5 & $<0.001$ \\
\hline \multicolumn{4}{|l|}{ Duración de la estancia (\%) / Length of stay (\%) } \\
\hline 1-7 dias / 1-7 days & 52.3 & 77.5 & \\
\hline 8-14 dias / 8-14 days & 43.8 & 18.9 & \\
\hline Más de 14 dias / Over 14 days & 4.0 & 3.6 & $<0.001$ \\
\hline \multicolumn{4}{|l|}{ Razones para elegir destino vacacional (\%) / Reasons for choosing holiday destination (\%) } \\
\hline Coste / Cost & 17.5 & 7.4 & $<0.001$ \\
\hline Vida nocturna / Nightlife & 82.9 & 89.0 & $<0.01$ \\
\hline Cultura / Culture & 4.8 & 13.3 & $<0.001$ \\
\hline Clima / Weather & 55.5 & 43.4 & $<0.001$ \\
\hline Trabajo / Work & 1.8 & 3.4 & NS \\
\hline Visitar familia/amigos / Visiting family/friends & 8.3 & 9.9 & NS \\
\hline \multicolumn{4}{|l|}{ Consumo de sustancias en Gran Bretaña $(\%)^{2} \mid$ Substance use in the UK $(\%)^{2}$} \\
\hline Alcohol / Alcohol & 97.8 & 98.1 & NS \\
\hline Tabaco / Tobacco & 34.7 & 40.8 & $<0.05$ \\
\hline Cannabis / Cannabis & 13.7 & 19.4 & $<0.05$ \\
\hline Exxtasis / Ecstasy & 7.3 & 33.5 & $<0.001$ \\
\hline Cocaina / Cocaine & 9.3 & 33.7 & $<0.001$ \\
\hline Anfetamina / Amphetamine & 1.6 & 4.2 & $<0.05$ \\
\hline Ketamina / Ketamine & 1.8 & 7.8 & $<0.001$ \\
\hline $\mathrm{GHB} / \mathrm{GHB}$ & 1.2 & 1.5 & NS \\
\hline
\end{tabular}

P: Relevancia de la diferencia entre Gran Bretaña. y en vacaciones / P: Significance of difference between home and on holiday

NS: No significativo / NS: Not significant

${ }^{1}$ : Números que no suman el 100\% debido al redondeo / ${ }^{\prime}$ : Numbers may not add up to $100 \%$ due to rounding

2: Individuos que han consumido en los últimos 12 meses $/{ }^{2}$ : Individuals who had used in the last 12 months

de los visitantes de Mallorca declararon haber participado en una pelea durante las vacaciones, frente al 2,9\% de los visitantes de Ibiza $(p<0.001)$. En Mallorca los hombres fueron mucho más propensos que las mujeres a vivir experiencias negativas en vacaciones (discusiones, peleas, daños no intencionados y enfermedades); en lbiza las diferencias de género se limitaron a la participación en peleas.

La mayoría de turistas en ambos destinos viajaron sin pareja sexual (88,4\% Mallorca, 73,8\% Ibiza, $p<0.001$; Tabla 2). Entre los que viajaron sin pareja, el $29,8 \%$ en Mallorca y el $25,3 \%$ en Ibiza mantuvieron relaciones sexuales durante las vacaciones; casi uno de cada cinco en Mallorca y uno de cada ocho en lbiza mantuvieron relaciones con más de una persona. En ambos lugares la mayoría de los que mantuvieron relaciones con al menos una persona utilizaron condones sistemáticamente. Sin embargo, un tercio mantuvieron relaciones sin preservativo, y uno de cada seis mantuvieron relaciones sin protección con más de una persona. Los hombres tuvieron más parejas sexuales y practicaron más a menudo sexo sin protección.

Para analizar las diferencias entre los niveles de consumo de sustancias en el país de origen y en vacaciones, se midieron los cambios de frecuencia en el consumo de cinco de las sustancias más habituales: alcohol, tabaco, cannabis, éxtasis y cocaína (análisis limitado a los que consumieron alguna de esas sustancias en ambos destinos; Tabla 3). En ambos casos la frecuencia del consumo de todas las sustancias aumentó en vacaciones. Por ejemplo, mientras sólo el 2,4\% de los consumidores de éxtasis en la muestra de lbiza admitieron and needing to see a doctor/go to hospital were more commonly reported by the Majorca sample. For example, $11.6 \%$ of those visiting Majorca reported having been involved in a fight during their holiday compared with $2.9 \%$ of those visiting Ibiza $(p<0.001)$. In Majorca, males were significantly more likely than females to report negative holiday outcomes (arguments, fighting, unintentional injury and illness); in lbiza gender differences were limited to involvement in fights.

The majority of visitors to both destinations were travelling without a sexual partner, (88.4\% Majorca, $73.8 \%$ lbiza, $\mathrm{P}<0.001$; Table 2). Of those travelling without a sexual partner, 29.8\% in Majorca and 25.3\% in Ibiza reported having had sex during their stay; almost one in five in Majorca and one in eight in lbiza reported sex with more than one new partner. In both locations, the majority of those having sex with at least one new partner reported consistently using condoms. However a third of those having sex with a new partner reported doing so without using a condom, and one in six reported unprotected sex with more than one new partner. Males were more likely to report multiple sexual partners and unprotected sex.

To explore how levels of substance use differed between home and holiday use, changes in frequency of use of the five most commonly used substances (alcohol, tobacco, cannabis, ecstasy and cocaine) at home and on holiday were measured (limited to those using each substance in both locations; Table 3). For both samples, frequency of use of all 
Tabla 2: Conducta de riesgo en vacaciones, según el lugar del estudio

Table 2: Risk behaviour during the holiday, by survey location

\begin{tabular}{|c|c|c|c|c|c|c|c|c|c|}
\hline & \multicolumn{8}{|c|}{ Lugar del estudio / Location of survey } & \multirow[b]{3}{*}{ pt } \\
\hline & \multicolumn{4}{|c|}{ Mallorca / Majorca } & \multicolumn{4}{|c|}{ Ibiza } & \\
\hline & Total & Hombre/Male & Mujer/Female & $p^{*}$ & Total & Hombre/Male & Mujer/Female & $p^{*}$ & \\
\hline \multicolumn{10}{|l|}{$\begin{array}{l}\text { Consumo de sustancias en vacaciones }(\%) / \\
\text { Using substances during the holiday }(\%)\end{array}$} \\
\hline Alcohol / Alcohol & 99.4 & 100.0 & 98.8 & NS & 98.7 & 99.2 & 98.3 & NS & NS \\
\hline Tabaco / Tobacco & 39.4 & 39.8 & 39.0 & NS & 47.1 & 46.2 & 47.9 & NS & $<0.05$ \\
\hline Cannabis / Cannabis & 9.5 & 16.3 & 3.1 & $<0.001$ & 20.1 & 26.9 & 14.5 & $<0.001$ & $<0.001$ \\
\hline Ėxtasis / Ecstasy & 4 & 4.5 & 3.5 & NS & 44.2 & 55.5 & 34.9 & $<0.001$ & $<0.001$ \\
\hline Cocaina / Cocaine & 7.5 & 10.6 & 4.6 & $<0.05$ & 34.2 & 42.9 & 27.0 & $<0.001$ & $<0.001$ \\
\hline Anfetamina / Amphetamine & 1.8 & 2.8 & 0.8 & NS & 5.1 & 5.9 & 4.5 & NS & $<0.005$ \\
\hline Ketamina / Ketamine & 1.6 & 2.4 & 0.8 & NS & 14.2 & 18.5 & 10.7 & $<0.05$ & $<0.001$ \\
\hline $\mathrm{GHB} / \mathrm{GHB}$ & 1.8 & 2.8 & 0.8 & NS & 3.6 & 2.9 & 4.2 & NS & NS \\
\hline \multicolumn{10}{|l|}{$\begin{array}{l}\text { Consumo drogas ilegales en vacaciones B }(\%) \text { / } \\
\text { Illicit drugs used on holiday B (\%) }\end{array}$} \\
\hline Ninguna / None & 86.1 & 77.6 & 94.2 & & 46.1 & 33.6 & 56.4 & & \\
\hline & 8.5 & 15.4 & 1.9 & & 18.6 & 21.4 & 16.3 & & \\
\hline $2-3$ & 3.8 & 4.5 & 3.1 & & 26.4 & 33.6 & 20.4 & & \\
\hline 4 o más / 4 or more & 1.6 & 2.4 & 0.8 & $<0.001$ & 8.9 & 11.3 & 6.9 & $<0.001$ & $<0.001$ \\
\hline \multicolumn{10}{|l|}{$\begin{array}{l}\text { Frecuencia de embriaguez en vacaciones }(\%) \\
\text { Frequency of drunkenness during holiday }(\%)\end{array}$} \\
\hline 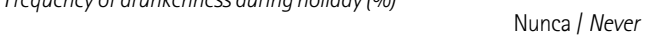 & 4.9 & 3.0 & 6.7 & & 10.3 & 7.9 & 12.3 & & \\
\hline Una vez a la semana o menos / Once a week or less & 8 & 2.1 & 13.4 & & 7.9 & 3.9 & 11.2 & & \\
\hline 2-4 dias a la semana / 2-4 days a week & 26.3 & 18.6 & 33.6 & & 28.7 & 22.7 & 33.6 & & \\
\hline $5+$ dias a la semana / 5+ days a week & 60.8 & 76.4 & 46.2 & $<0.001$ & 53.2 & 65.6 & 43.0 & $<0.001$ & $<0.01$ \\
\hline \multicolumn{10}{|l|}{$\begin{array}{l}\text { Experiencias negativas en vacaciones }(\%) / \\
\text { Reporting negative holiday experiences }(\%)\end{array}$} \\
\hline Participación en una pelea / Involved in a fight & 11.6 & 20.6 & 3.1 & $<0.001$ & 2.9 & 4.8 & 1.4 & $<0.05$ & $<0.001$ \\
\hline Participación en una discusión / Involved in an argument & 28.2 & 38.8 & 18.2 & $<0.001$ & 11.6 & 13.9 & 9.7 & NS & $<0.001$ \\
\hline Heridas por accidentes / Injured in an accident & 12.8 & 16.5 & 9.3 & $<0.05$ & 7.7 & 10.0 & 5.9 & NS & $<0.01$ \\
\hline Enfermedad / IIIness & 56 & 63.5 & 48.8 & $<0.005$ & 41.9 & 39.6 & 43.8 & NS & $<0.001$ \\
\hline Visita al medico/hospital / Had to go see a doctor/go to hospital & 8.4 & 8.6 & 8.2 & NS & 4.4 & 4.3 & 4.5 & NS & $<0.05$ \\
\hline Robo de pertenencias / Had belongings stolen & 10 & 11.1 & 8.9 & NS & 6.7 & 5.6 & 7.6 & NS & NS \\
\hline Viajan con pareja sexual (\%) / Travelling with a sexual partner (\%) & 11.6 & 9.5 & 13.6 & NS & 26.2 & 28.6 & 24.3 & NS & $<0.001$ \\
\hline \multicolumn{10}{|l|}{ Viajan sin pareja sexual: / Of those travelling without a sexual partner: } \\
\hline \multicolumn{10}{|l|}{$\begin{array}{l}\text { Número de parejas sexuales en vacaciones }(\%) \\
\text { Number of sexual partners on holiday }(\%)\end{array}$} \\
\hline 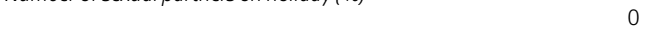 & 70.2 & 57.5 & 82.0 & & 74.7 & 69.1 & 78.8 & & \\
\hline 1 & 12.0 & 14.0 & 10.2 & & 13.4 & 11.5 & 14.8 & & \\
\hline 2 a $4 / 2$ to 4 & $\begin{array}{l}11.0 \\
68\end{array}$ & $\begin{array}{l}15.5 \\
130\end{array}$ & $\begin{array}{l}6.8 \\
10\end{array}$ & $<0001$ & $\begin{array}{l}8.2 \\
3.7\end{array}$ & $\begin{array}{l}15.1 \\
43\end{array}$ & $\begin{array}{l}3.2 \\
32\end{array}$ & $<0.005$ & NS \\
\hline \multicolumn{10}{|l|}{$\begin{array}{l}\text { Viajan sin pareja sexual y practican sexo en vacaciones: / Of those } \\
\text { travelling without a sexual partner and having sex during the holiday }\end{array}$} \\
\hline \multicolumn{10}{|l|}{$\begin{array}{l}\text { Número de relaciones sexuales sin protección }(\%) \text { / } \\
\text { Number of unprotected sexual partners }(\%)\end{array}$} \\
\hline 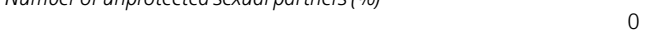 & 66.4 & 62.2 & 75.7 & & 65.1 & 58.1 & 72.5 & & \\
\hline 1 & 17.6 & 17.1 & 18.9 & & 19.3 & 20.9 & 17.5 & & \\
\hline 2 a $4 / 2$ to 4 & 7.6 & 8.5 & 5.4 & & 6.0 & 9.3 & 2.5 & & \\
\hline $5+$ & 8.4 & 12.2 & 0.0 & NS & 9.6 & 11.6 & 7.5 & NS & NS \\
\hline
\end{tabular}

P: Entre genero sin localización; t: P Entre totales por localización; $\beta$ : Drogas ilegales analizadas: cannabis, éxtasis, cocaina, anfetamina, ketamina y GHB. NS: No significativo.

p: Between genders within location; + : p Between location totals; $\beta$ : Illicit drugs surveyed were: cannabis, ecstasy, cocaine, amphetamine, ketamine and GHB NS: Not significant

haber consumido la droga dos o más veces por semana en Gran Bretaña, durante las vacaciones nueve de cada diez la consumieron con esa frecuencia, y el $46,7 \%$ la consumieron cinco o más días a la semana.

Por último, la regresión logística se utilizó para identificar factores independientemente asociados con los resultados de las vacaciones (participación en peleas, daño no intencionado, relaciones sexuales con una nueva pareja, múltiples parejas sexuales, y sexo sin protección; Tabla 4). Los análisis de las consecuencias sexuales se estudian sólo en los que llegaron al destino vacacional sin pareja, y los análisis de la conducta sexual de riesgo a los que llegaron sin pareja y mantuvieron relaciones. El lugar de la encuesta no se asoció con ninguna consecuencia. Sin embargo, los hombres substances increased during the holiday. For example, while just $2.4 \%$ of ecstasy users in the lbiza sample reported using the drug two or more times per week at home, during the holiday nine out of ten users reported use at this frequency, with $46.7 \%$ reporting use five or more days per week.

Finally, logistic regression was used to identify factors independently associated with selected holiday outcomes (being involved in a physical fight, unintentional injury, sex with a new partner, multiple sexual partners, and unprotected sex; Table 4). Analyses of sexual outcomes were limited to those arriving without a partner, and analyses of risky sexual behaviour limited to those arriving without a partner and having sex. Results found location of survey was not associated with any outcome. However being male 
Tabla 3: Frecuencia del consumo de sustancias en Gran Bretaña y en el extranjero (limitado a los que consumieron sustancias individuales en ambas ubicaciones) Table 3: frequency of substance use at home and abroad (limited to those using individual substances in both locations)

3a) Mallorca / 3a) Majorca

\begin{tabular}{|c|c|c|c|c|c|c|c|}
\hline & & \multirow[b]{2}{*}{$n$} & \multicolumn{4}{|c|}{ Frecuencia de consumo (\%) / Frequency of use (\%) } & \multirow[b]{2}{*}{$P^{*}$} \\
\hline & & & $\begin{array}{l}<1 \text { vez semana / } \\
<\text { Once a week }\end{array}$ & $\begin{array}{l}1 \text { vez semana / } \\
\text { Once a week }\end{array}$ & $\begin{array}{l}\text { 2-4 dias semana / } \\
\text { 2-4 days a week }\end{array}$ & $\begin{array}{l}5+\text { dias semana / } \\
5+\text { days a week }\end{array}$ & \\
\hline \multirow[t]{2}{*}{ Alcohol / Alcohol } & Gran Bretaña / Home & \multirow[t]{2}{*}{491} & 21.6 & 41.1 & 29.7 & 7.5 & \\
\hline & Mallorca / Majorca & & 0.8 & 0.6 & 8.8 & 89.8 & $<0.001$ \\
\hline \multirow[t]{2}{*}{ Tabaco / Tobacco } & Gran Bretaña / Home & \multirow[t]{2}{*}{166} & 22.3 & 9.0 & 10.8 & 57.8 & \\
\hline & Mallorca / Majorca & & 3.0 & 6.0 & 17.5 & 73.5 & $<0.001$ \\
\hline \multirow[t]{2}{*}{ Cannabis / Cannabis } & Gran Bretaña / Home & \multirow{2}{*}{30} & 43.3 & 6.7 & 16.7 & 33.3 & \\
\hline & Mallorca / Majorca & & 13.3 & 23.3 & 36.7 & 26.7 & $<0.05$ \\
\hline \multirow[t]{2}{*}{ Éxtasis / Ecstasy } & Gran Bretaña / Home & \multirow{2}{*}{15} & 66.7 & 13.3 & 6.7 & 13.3 & \\
\hline & Mallorca / Majorca & & 33.3 & 20.0 & 13.3 & 33.3 & NS \\
\hline \multirow[t]{2}{*}{ Cocaina / Cocaine } & Gran Bretaña / Home & \multirow[t]{2}{*}{25} & 72.0 & 12.0 & 8.0 & 8.0 & \\
\hline & Mallorca / Majorca & & 32.0 & 24.0 & 12.0 & 32.0 & $<0.05$ \\
\hline
\end{tabular}

3b) Ibiza

\begin{tabular}{|c|c|c|c|c|c|c|c|}
\hline & & \multirow[b]{2}{*}{$\mathrm{n}$} & \multicolumn{4}{|c|}{ Frecuencia de consumo (\%) / Frequency of use (\%) } & \multirow[b]{2}{*}{$p^{*}$} \\
\hline & & & $\begin{array}{c}<1 \text { vez semana / } \\
<\text { Once a week }\end{array}$ & $\begin{array}{c}1 \text { vez semana / } \\
\text { Once a week }\end{array}$ & $\begin{array}{l}\text { 2-4 dias semana / } \\
\text { 2-4 days a week }\end{array}$ & $\begin{array}{l}5+\text { dias semana / } \\
5+\text { days a week }\end{array}$ & \\
\hline \multirow[t]{2}{*}{ Alcohol / Alcohol } & Gran Bretaña / Home & 514 & 18.5 & 36.6 & 38.3 & 6.6 & \\
\hline & Ibiza & & 1.2 & 1.0 & 11.5 & 86.4 & $<0.001$ \\
\hline \multirow[t]{2}{*}{ Tabaco / Tobacco } & Gran Bretaña / Home & 206 & 12.6 & 11.7 & 22.3 & 53.4 & \\
\hline & lbiza & 200 & 1.5 & 1.0 & 9.7 & 87.9 & $<0.001$ \\
\hline \multirow[t]{2}{*}{ Cannabis / Cannabis } & Gran Bretaña / Home & 65 & 29.2 & 18.5 & 23.1 & 29.2 & \\
\hline & Ibiza & & 7.7 & 15.4 & 27.7 & 49.2 & $<0.01$ \\
\hline \multirow[t]{2}{*}{ Éxtasis / Ecstasy } & Gran Bretaña / Home & 167 & 83.2 & 12.6 & 2.4 & 1.8 & \\
\hline & Ibiza & & 0.6 & 10.2 & 42.5 & 46.7 & $<0.001$ \\
\hline \multirow[t]{2}{*}{ Cocaina / Cocaine } & Gran Bretaña / Home & 144 & 74.3 & 16.7 & 5.6 & 3.5 & \\
\hline & Ibiza & & 13.2 & 18.1 & 41.7 & 27.1 & $<0.001$ \\
\hline
\end{tabular}

P: Relevancia de la diferencia entre la frecuencia de consumo en el país de origen y durante las vacaciones. NS: No significativo /

P: Significance of difference between frequency of use at home and on holiday. NS: Not significant

Tabla 4: Cociente ajustado de posibilidades de participación en peleas, daños no intencionados y conducta sexual de riesgo en vacaciones. Table 4: Adjusted odds ratios for involvement in physical fighting, unintentional injury and risky sexual behaviour on holiday

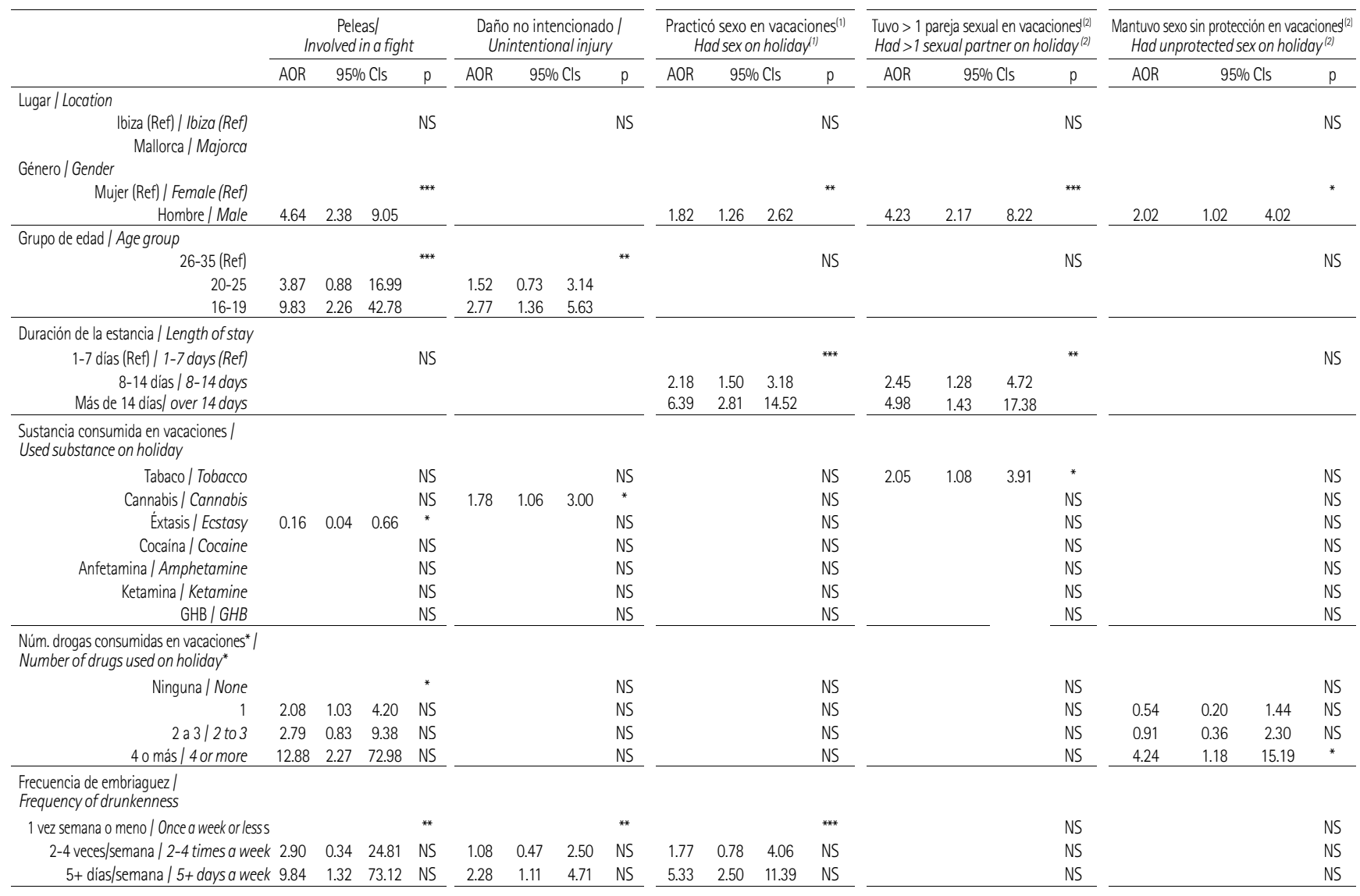

1: Limitado a los que viajaron sin pareja sexual. 2: Limitado a los que viajaron sin pareja sexual y practicaron sexo en vacaciones.

AOR: Cociente Ajustado de Posibilidades; $95 \%$ Cls, 95\% Intervalos de confianza. La significación se muestra como ${ }^{*} \mathrm{p} 0.05,{ }^{* *} \mathrm{p} 0.01{ }_{1}{ }^{* * *} \mathrm{p}<.001$. La estadistica utiliza un análisis de regresión logistica condicional eliminando del modelo las variables no significativas ( $\mathrm{p} \geq 0.05$ ) y, en consecuencia, recalculando el modelo. Las categorías de referencia para cada variable se identifican con (Ref).

1: Limited to those arriving without a sexual partner. 2: Limited to those arriving without a sexual partner and having sex on holiday.

AOR: Adjusted Odds Ratios; $95 \% \mathrm{Cls}, 95 \%$ confidence intervals. Significance is shown as ${ }^{*} P<0.05,{ }^{* *} P<0.01,{ }^{* * *} P<0.001$. Statistics utilise a stepwise backward conditional logistic regression analysis with variables that are not significant $(P \geq 0.05)$ being removed from the model and subsequently the model recalculated. Reference categories for each variable are identified with (Ref). 
fueron más propensos a todos los resultados, exceptuando el daño no intencionado. En particular, las probabilidades de participar en peleas y tener múltiples parejas sexuales fueron cuatro veces más altas entre los hombres que entre las mujeres. Ser joven se asoció estrechamente a las agresiones físicas y al daño no intencionado, mientras que las estancias de mayor duración correspondieron a las relaciones sexuales con parejas nuevas y con múltiples parejas. La frecuencia de la embriaguez tuvo mucho que ver con las peleas, el daño no intencionado y las relaciones sexuales con una pareja nueva. El consumo de tabaco en vacaciones se vinculó a las relaciones con múltiples parejas, mientras que el de cannabis aparece asociado al daño no intencionado, y el de éxtasis al riesgo reducido de participación en peleas. El policonsumo de drogas (consumo de más de un tipo de droga ilegal en vacaciones) se asoció a la violencia, mientras que el consumo de cuatro o más drogas en vacaciones se asoció al sexo sin protección.

\section{DISCUSIÓN}

En lo referente a la encuesta sobre conductas conflictivas o ilegales (p.ej., consumo de drogas), nuestro estudio puede estar condicionado por la ocultación o la exageración del consumo de sustancias, la actividad sexual, la violencia y otros aspectos. El lugar del estudio potencia estos efectos, pues los participantes cubrieron los cuestionarios en las filas de facturación del aeropuerto (zonas de privacidad limitada) antes de pasar los controles de seguridad. Para reducir estos efectos utilizamos una metodología establecida que informaba a los participantes del propósito del estudio, les garantizaba la confidencialidad y les proporcionaba un mecanismo de participación totalmente anónimo. Y así, no se les pidió información identificable, se les ofrecieron tablillas para que cubriesen los cuestionarios con comodidad y confidencialidad, y los cuestionarios se entregaron acto seguido a los encuestadores en sobres cerrados y sin marcas antes de que los participantes facturasen sus vuelos. Este sistema tiene varias ventajas: la mayoría de los turistas británicos que van a Mallorca e lbiza viajan en avión, muchas veces tienen que esperar bastante tiempo para facturar, y los participantes están en condiciones de ofrecer información sobre las vacaciones, pues las tienen aún en mente. La embriaguez fue definida por los propios encuestados y no se preguntó por las cantidades de alcohol consumido. Sin embargo, puesto que los efectos del alcohol en los individuos dependen de factores como el estilo de consumo, la altura, el peso y la ingesta de comida, este aspecto proporcionó una medida subjetiva del nivel de consumo de alcohol que según los participantes los había conducido a la embriaguez. Por último, la selección fue oportunista y se realizó siguiendo un criterio de conveniencia. Sin embargo, obtuvimos una alta participación utilizando un método para abordar a todos los posibles participantes, con resultados que se consideran ampliamente representativos de los turistas que visitan ambas islas.

Los resultados indican que a los jóvenes turistas británicos les atraen Mallorca e lbiza por su vida nocturna. La participación habitual en la vida nocturna en Gran Bretaña se asocia was linked to all outcomes apart from unintentional injury. In particular, odds of involvement in fights and having multiple sexual partners were four times higher for males than females. Being young was strongly associated with physical fighting and unintentional injury, while longer length of stay was associated with having sex with a new partner and multiple sexual partners. Frequency of drunkenness was strongly associated with fighting, unintentional injury and sex with a new partner. Using tobacco on holiday was associated with having multiple sexual partners whilst using cannabis was associated with unintentional injury; ecstasy use was associated with reduced risk of involvement in fighting. Polydrug use (using more than one type of illicit drug on holiday) was associated with violence, whilst using four or more drugs during the holiday was also associated with having unprotected sex.

\section{DISCUSSION}

As with all surveys of behaviours that are sensitive and even illegal (e.g. drug use), our study may have been influenced by under-reporting or exaggeration of substance use, sexual activity, violence and other outcomes. Survey location may have exacerbated these effects, with participants completing questionnaires in airport check-in queues (areas with limited private space) prior to passing through security controls. To reduce these effects we used an established methodology that ensured participants were informed of the survey's purpose, assured of its confidentiality, and provided with a clearly anonymous mechanism of participation. Thus no identifiable information was requested, clipboards were provided to facilitate completion and confidentiality, and questionnaires were returned immediately to researchers in unmarked, sealed envelopes prior to participants checking in to flights. The benefits of this approach include: most British holidaymakers to Majorca and Ibiza travel by air; there are often long waits for check-in; and participants are able to provide information covering the entire holiday period whilst this is fresh in their minds. Drunkenness was self-defined and provided no detail on actual quantities of alcohol consumed. However, with the effects of alcohol on individuals being influenced by factors such as drinking pattern, height, weight and food consumption, this did provide a subjective measure of a level of alcohol consumption that participants believed had caused them to become drunk. Finally, recruitment was opportunistic and conducted on a convenience basis. However, we achieved high compliance using a method that approached all potential participants, with findings intended to be broadly representative of holidaymakers visiting each island.

Findings suggest that young British holidaymakers are attracted to both Majorca and Ibiza through their reputation for nightlife. Regular participation in nightlife in the UK is associated with higher levels of substance use, ${ }^{4}$ 
con elevados niveles de consumo de sustancias ${ }^{4}$, y en consecuencia hay más consumidores de sustancias entre los que eligen pasar las vacaciones en centros turísticos orientados a la vida nocturna que entre los jóvenes en general. No obstante, los que eligieron Ibiza eran más mayores y había más consumidores de drogas ilegales que entre los que eligieron Mallorca (Tabla 1), y la mayoría de consumidores de drogas en Ibiza afirmaron haber consumido más de una droga durante su estancia. El éxtasis fue la droga más consumida, seguido por la cocaína y el cannabis. Este orden de consumo de drogas en lbiza contrasta con el registrado en Gran Bretaña entre la población en general, pues en el segundo caso el cannabis es la droga más consumida $(17,9 \%$ de los jóvenes entre 12 y 24 años el año pasado), seguido por la cocaína $(5,0 \%)$ y el éxtasis $(3,9 \%) .{ }^{4}$ Estas tendencias nacionales se reflejaron en la muestra de Mallorca. Los niveles superiores de consumo de drogas (dominado por el éxtasis) en lbiza constatan la atracción que ejerce la isla sobre una población acostumbrada al consumo de drogas y habitual de los clubes ${ }^{30-32}$ e indican gran facilidad para conseguir drogas en Ibiza. En Ibiza la prevalencia del consumo de cada droga es más alta en vacaciones que en Gran Bretaña, lo cual apunta a la experimentación de drogas nuevas y a un mayor consumo de drogas en el período vacacional (datos recogidos en ambos lugares) ${ }^{29}$. Por tanto Ibiza debe considerarse un lugar primordial para ofrecer información e imponer medidas de reducción del daño provocado por el consumo de drogas.

Tanto en lbiza como en Mallorca los individuos que consumieron drogas y alcohol lo hicieron con mayor frecuencia en vacaciones que en Gran Bretaña. Más de la mitad de turistas de ambos destinos se embriagaron cinco o más noches por semana durante sus vacaciones (Tabla 2). El consumo de alcohol y de drogas supone un grave riesgo para la salud a corto y largo plazo, agravado por el consumo nocturno excesivo. Durante la temporada turística de 2005 en el hospital Ca'n Misses de lbiza se trataron 135 casos graves de intoxicación por drogas sólo en el mes de agosto; el 80\% eran hombres no españoles entre 20 y 29 años, y la mayoría sufrieron la intoxicación en los night-clubs de la isla ${ }^{33}$. Un estudio en detalle de los casos de urgencia con sintomas de intoxicación en lbiza demuestra que la mayoría habían realizado policonsumo de drogas, casi siempre éxtasis mezclado con sustancias como alcohol, cocaina y ketamina ${ }^{20}$. No se ha medido el impacto del consumo de alcohol por parte de los turistas en los servicios sanitarios de los centros turísticos españoles, pero los ingresos hospitalarios y las muertes relacionadas con el alcohol en Gran Bretaña van en aumento ${ }^{34,35}$. A largo plazo el consumo excesivo de alcohol se asocia con una amplia gama de enfermedades como patologias hepáticas, arritmias cardíacas y cáncer ${ }^{36}$. Sin embargo, los efectos graves de la intoxicación etílica son mucho más visibles, y entre ellos se cuentan los vómitos, el daño no intencionado, las agresiones y la conducta antisocia| ${ }^{37-41}$

Entre los visitantes de Mallorca se registraron casos de embriaguez más frecuentes y consecuencias más negativas que en lbiza (Tabla 2). Más de la mitad de los que escogieron Mallorca se pusieron enfermos durante su estancia, un tercio participaron en discusiones y uno de cada diez en una pelea and consequently those choosing to holiday in nightlifefocused resorts are likely to be higher substance users than young people in the general population. However, those choosing to visit lbiza were older and significantly more likely to report illicit drug use than those choosing Majorca (Table 1), with the majority of drug users in Ibiza reporting using more than one drug during their stay. Ecstasy was the most commonly reported drug used, followed by cocaine and cannabis. This order of drug use in Ibiza contrasts with that seen in the UK amongst the general population, where cannabis is the most commonly used drug (17.9\% of $16-24$ year olds in the last year), followed by cocaine (5.0\%) and ecstasy (3.9\%). ${ }^{4}$ These national trends were reflected in the Majorca sample. The higher levels of drug use (dominated by ecstasy) in Ibiza demonstrate the island's appeal to a drug-experienced clubbing population ${ }^{30-32}$ and suggest widespread availability of drugs in Ibiza. In Ibiza, reported prevalence of use of each drug is higher on holiday than at home, showing experimentation with new drugs and relapse into drug use during the holiday period (data reported elsewhere). ${ }^{29}$ Thus Ibiza should be considered a critical location for targeting information and harm reduction measures regarding drug use.

In both Ibiza and Majorca, individuals who used drugs and alcohol did so more frequently on holiday than they would normally at home. Further, over half of holidaymakers to both destinations reported being drunk on five or more nights per week during their holiday (Table 2). Both alcohol and drug use carry significant risks to short and long term health, which are likely to be exacerbated by excessive, nightly use. During the 2005 tourist season, 135 serious drug poisonings were treated at Ibiza's Can Misses hospital in August alone; $80 \%$ were non-Spanish males in their 20s, and most were brought in from the island's nightclubs. ${ }^{33}$ More detailed study of emergency presentations for toxic symptoms in lbiza found most involved polydrug use, typically ecstasy mixed with substances including alcohol, cocaine and ketamine. ${ }^{20}$ Whilst the impacts of tourists' alcohol use on health services in Spanish resorts has not been measured, both hospital admissions and deaths related to alcohol in the UK are increasing. ${ }^{34,35}$ In the long-term, alcohol misuse is associated with a wide range of health conditions including liver disease, cardiac arrhythmias and cancers. ${ }^{36}$ However the acute effects of alcohol intoxication are far more visible, and include vomiting, unintentional injury, aggression and antisocial behaviour. ${ }^{37-41}$

Visitors to Majorca reported both more frequent drunkenness and more negative outcomes than those to lbiza (Table 2). Over half in Majorca reported having been ill during their stay, around a third were involved in an argument, and over one in ten in a fight or an accident resulting in injury. Such problems are likely to cause a significant burden to health and criminal justice services in holiday resorts. In our survey over $8 \%$ of both males and females in Majorca 
o en un accidente con resultado de heridos. Estos problemas suponen una sobrecarga significativa para los servicios de orden público de los centros turísticos. En nuestro estudio en torno al $8 \%$ de los hombres y mujeres que visitaron Mallorca acudieron al médico o al hospital durante las vacaciones. A pesar de la carga de violencia y agresiones soportada por Mallorca y tras controlar los factores de confusión, el lugar del estudio no se asoció con peleas ni con heridas derivadas de accidentes. Ambos factores se asociaron en gran medida con la embriaguez frecuente. Las peleas también se asociaron a los jóvenes, hombres y policonsumidores de drogas; aunque el éxtasis redujo las posibilidades de pelear. Esto coincide con lo observado en otras partes ${ }^{42}$ y apoya la fama del éxtasis como droga de efectos socializadores. Los estudios dedicados a las funciones del consumo de éxtasis demuestran que los consumidores valoran en dicha droga efectos como la promoción de la empatía, el relajamiento de las defensas personales y el disfrute de la compañía de otras personas ${ }^{43}$.

En Mallorca preocupan especialmente los niveles de violencia. Una serie de investigaciones asocian la violencia con la elevada densidad de lugares de venta de alcohol ${ }^{44}$, niveles altos de embriaguez ${ }^{45}$, y establecimientos mal gestionados con actitudes permisivas ante la bebida ${ }^{46}$. Estos factores constituyen características comunes de los centros turísticos que reciben a turistas jóvenes. En Gran Bretaña las fiestas de fin de semana en los ambientes nocturnos de los centros de los pueblos y ciudades se asocian desde hace mucho tiempo a la violencia, y por ello se ha invertido mucho en medidas para prevenir el consumo de alcohol excesivo, mejorar la gestión de bares y night-clubs y crear entornos de vida nocturna más seguros ${ }^{47}$. Esas medidas consumen muchos recursos; por ejemplo, requieren una presencia policial significativa durante las noches de los fines de semana, y los impactos de las fiestas vacacionales recargan las tareas de las autoridades locales. En Mallorca (p. ej., en el centro de Magalluf orientado a la juventud) se han tomado medidas significativas para organizar la vida nocturna y prevenir la violencia en los centros turísticos ${ }^{48}$, una necesidad dado lo mucho que perjudica al turismo la fama de violencia ${ }^{20,21}$. Sin embargo, hay menos problemas de embriaguez y violencia en las conductas nocturnas de la juventud española ${ }^{28}$. Por tanto, es necesaria la colaboración entre las autoridades de los centros turísticos y las de los países de origen de los turistas para comparar experiencias sobre la organización de la conducta y la vida nocturna de los jóvenes. Aparte de facilitar la creación de ambientes nocturnos más seguros en los centros turísticos, dicho intercambio permite a las culturas con elevados niveles de embriaguez y violencia entre los jóvenes aprender de las culturas con vida nocturna menos problemática y contribuye a evitar culturas de vida nocturna nociva como las que se desarrollan en los países en los que el consumo de alcohol entre los jóvenes va en aumento.

A pesar de las diferencias de consumo de drogas entre las muestras de Mallorca e lbiza, la embriaguez y consecuencias como la violencia y los elevados niveles de conducta sexual de riesgo fueron similares en ambos lugares. Dos tercios de los que practicaron sexo con al menos otra persona, utilizaron condones sistemáticamente. Sin embargo, un tercio reported having visited a doctor or gone to hospital during their holiday. Despite the burden of violence and injuries falling on Majorca, after controlling for confounding factors location of survey was not associated to either fighting or accidents resulting in injury. Both however were strongly associated with frequency of drunkenness. Fighting was also associated with being young, male, and a polydrug user; although ecstasy use reduced odds of fighting. This is consistent with findings elsewhere ${ }^{42}$ and supports ecstasy's long-held reputation as a drug with prosocial effects. Studies exploring the functions of ecstasy use have shown the drug to be valued by users for effects including the promotion of empathy, lowered personal defences, and enjoyment of the company of others. ${ }^{43}$

Levels of violence in Majorca are a particular concern. A range of research links violence to high densities of drinking premises, ${ }^{44}$ high levels of drunkenness, ${ }^{45}$ and poorly managed, permissive drinking settings. ${ }^{46}$ Such factors can be common features of holiday resorts that cater for young visitors. In the UK, weekend partying in town and city centre nightlife environments has long been linked to violence and vast resources have been channelled into measures to prevent excessive drinking, improve management of bars and nightclubs and create safer nightlife environments. ${ }^{47}$ Such measures are resource intensive, for example involving significant police presence in nightlife on weekend nights. In holiday resorts, every night can be a weekend night and the impacts of holiday partying can place large demands on local authorities. Already in Majorca (e.g. the youth-focused resort of Magaluf), significant steps are being taken to manage nightlife and prevent violence in holiday resorts; ${ }^{48}$ a necessity given the harm a reputation for violence can place on tourism. ${ }^{20,21}$ However problems of drunkenness and violence feature less in the nightlife behaviours of Spanish youth. ${ }^{28}$ Thus, there is need for partnership between authorities in tourist resorts and those in tourists' countries of origins to share experiences of managing youth behaviour and nightlife. As well as facilitating the creation of safer nightlife in tourist resorts, such exchange should enable cultures with high youth drunkenness and violence to learn from those with less problematic nightlife cultures; and help prevent harmful nightlife cultures developing in countries were youth alcohol use is growing.

Despite differences between the Majorca and Ibiza samples in drug use, drunkenness and outcomes such as violence, reported levels of risky sexual behaviour were similar in both locations. Two thirds of those having sex with at least one new partner on holiday reported consistently using condoms. However a third reported unprotected sex with a new partner, with around $16 \%$ in both locations reporting unprotected sex with multiple partners. Having multiple sexual partners was associated with tobacco use, and having unprotected sex with using four or more drugs 
practicó sexo sin protección con una pareja nueva, y en torno al $16 \%$ en ambos lugares practicaron sexo con varias personas. La práctica de sexo con múltiples parejas se asoció al consumo de tabaco y el sexo sin protección al consumo de cuatro o más drogas durante las vacaciones. Dichas asociaciones muestran una concentración de conductas de riesgo en algunos individuos ${ }^{49}$. Por ejemplo, el perjuicio para la salud provocado por el tabaco es bien conocido, y los individuos que continúan fumando a pesar de conocer los riesgos que comporta tienen mayor tendencia a asumir riesgos en general, incluyendo ciertas conductas sexuales.

El sexo sin protección y tener parejas múltiples ponen a los individuos en riesgo de sufrir ITS, y cuando no se utilizan métodos de control de natalidad, de embarazos no deseados. Las tasas de ITS en Gran Bretaña aumentaron muchísimo en la última década: en el grupo de edad entre 16 y 24 años los nuevos diagnósticos de clamidia crecieron el 180\% entre 1998 y $2007^{6}$. Infecciones como la clamidia no muestran síntomas visibles, y por tanto la conducta sexual de riesgo en vacaciones contribuye a la propagación internacional de ITS cuando los individuos, al volver a su país de origen, mantienen relaciones sexuales. Los datos británicos indican que el $20 \%$ de los hombres y el $12 \%$ de las mujeres entre 16 y 24 años mantuvieron relaciones sexuales con una persona desconocida fuera de Gran Bretaña en los últimos cinco años ${ }^{50}$, mientras que en el grupo de edad entre 16 y 44 años casi la mitad de los que mantuvieron relaciones sexuales con una pareja nueva o no habitual no utilizaron condones ${ }^{51}$. Tras los periodos vacacionales se producen picos en la actividad de las clínicas que tratan enfermedades de transmisión sexual ${ }^{52}$. Por tanto, es prioritario fomentar la salud sexual en los centros turísticos para jóvenes.

Este estudio demuestra que los períodos vacacionales son épocas de excesos para los jóvenes turistas británicos, con elevado consumo de sustancias y altos niveles de daño no intencionado, violencia y conducta sexual de riesgo reconocidos en nuestra encuesta. Sin embargo, el comportamiento varía en los distintos centros turísticos, e identificar los centros que se asocian con determinados tipos de conductas de riesgo es fundamental para programar la reducción de daños y medidas de protección de la salud. Basándonos en nuestros resultados, lbiza debe considerarse un lugar prioritario para realizar intervenciones de control del consumo de drogas en particular, y Mallorca para el daño relacionado con el alcohol, incluyendo la violencia. En ambos lugares es necesario invertir en la promoción de la salud sexual. La ejecución de esas medidas es responsabilidad conjunta de las autoridades de los centros turísticos, que por un lado se benefician económicamente del turismo juvenil y por otro soportan los inconvenientes de los problemas de salud en vacaciones, y de las autoridades de los países de origen de los turistas, responsables de la protección de la salud de sus jóvenes.

\section{AGRADECIMIENTOS}

Agradecemos a las autoridades de los aeropuertos de Mallorca e lbiza su apoyo al proyecto; a Susanne Schnitzer, during the holiday. Such associations may show a clustering of risk behaviours in some individuals. ${ }^{49}$ For example, the damage to health associated with smoking is now widely understood and individuals who continue to smoke despite the known risks may be more likely to take risks in general, including with their sexual health.

Having unprotected sex and multiple sexual partners places individuals at risk of STIs, and where other methods of birth control are not used, unwanted pregnancy. Rates of STIs in the UK have increased dramatically in the last decade; in 16-24 year olds, new diagnoses of chlamydia increased by $180 \%$ between 1998 and $2007 .{ }^{6}$ With infections such as chlamydia often not having visible symptoms, risky sexual behaviour on holiday can contribute to the international spread of STIs as individuals returning from holiday engage in sexual relationships at home. British survey data show $20 \%$ of males and $12 \%$ of females aged 16-24 report sex with a new partner from outside the UK in the past five years, ${ }^{50}$ while across $16-44$ year olds, almost half of those having sex with any new or non-regular partner do not use condoms. ${ }^{51}$ Holiday periods are followed by peaks in attendance at sexual health clinics. ${ }^{52}$ Thus promoting sexual health in youth-focused holiday resorts should be a major priority.

This study has demonstrated that holiday periods are times of excess for young British holidaymakers, with elevated substance use and high levels of unintentional injury, violence and risky sexual behaviour reported across our sample. However behaviours vary between resorts and understanding which resorts are associated with which types of health risk behaviour is key in targeting harm reduction and health promotion measures. Based on our findings, lbiza should be regarded as a critical location for interventions addressing drug use in particular, and Majorca for those addressing alcohol-related harm, including violence. Both locations require investment in sexual health promotion. Implementing such measures should be seen as the joint responsibility of authorities in holiday resorts, which reap the economic benefits of youth tourism yet shoulder the burdens of holiday health problems, and those from tourists' home countries who are responsible for protecting the health of their young people.

\section{ACKNOWLEDGEMENTS}

We thank the airport authorities in Majorca and Ibiza for their support with the project; Susanne Schnitzer, Zara Anderson, Dan Hungerford, Encarna Roman, Margarida Ros, Joan Escandell, Daniela Volkel and Sylvia Nitschke for their assistance in data collection; Henry Edwards for his assis- 
Zara Anderson, Dan Hungerford, Encarna Román, Margarida Ros, Joan Escandell, Daniela Volkel y Sylvia Nitschke su labor de recogida de datos; a Henry Edwards su ayuda en el procesamiento de datos, y a todos los jóvenes turistas que participaron voluntariamente en esta encuesta. Agradecemos asimismo la colaboración de dos revisores anónimos que nos ayudaron a mejorar el borrador inicial de este artículo.

\section{Financiación}

Comisión Europea, Dirección General de Justicia, Libertad y Seguridad: Daphne 2006-1 (JLS/2006/Dap-1/069/ Y30CE-0117583/00-55).

Conflicto de intereses: Ninguno.

\section{REFERENCIAS / REFERENCES}

1. Currie $C$, Gabhainn $S N$, Godeau E et al (eds.). Inequalities in young people's health: international report from the HBSC 2005/06 survey. WHO policy series: health policy for children and adolescents, issue 5. Copenhagen: WHO Regional Office for Europe, 2008.

2. Hibell B, Andersson B, Bjarnasson T et al. The ESPAD report 2003: alcohol and other drug use among students in 35 European countries. Stockholm: The Swedish Council for Information on Alcohol and Other Drugs, 2004.

3. Hughes $K$, Anderson Z, Morleo M, Bellis MA. Alcohol, nightlife and violence: the relative contributions of drinking before and during nights out to negative health and criminal justice outcomes. Addiction 2008; 103: 60-5.

4. Hoare J and Flatley J. Drug misuse declared: findings from the 2007/08 British Crime Survey. London: Home Office, 2008.

5. Kershaw C, Nicholas S and Walker A. Crime in England and Wales 2007/08. London: Home Office, 2008.

6. Health Protection Agency. Selected STI diagnoses made at genitourinary medicine (GUM) clinics in the United Kingdom: 1998-2007. Available at: http://www.hpa.org.uk/web/ HPAweb\&HPAwebStandard/HPAweb_C/1195733775264. Accessed 31st October 2008.

7. Atkinson A, Anderson Z, Hughes K, Bellis MA, Sumnall $H$, Syed 0 . Interpersonal violence and illicit drugs. Liverpool: Centre for Public Health, Liverpool John Moores University, 2009.

8. Bellis MA, Hughes $K_{1}$ Calafat $A$, Juan M, Ramón A, Rodríguez JA, Mendes F, Schnitzer $S$ and Phillips-Howard P. Sexual uses of alcohol and drugs and the associated health risks: a cross sectional study of young people in nine European cities. BMC Public Health 2008; 8: 155.

9. HM Government. Safe. Sensible. Social. The next steps in the national alcohol strategy. London: HM Government, 2007.

10. HM Government. Drugs: protecting families and communities. The 2008 drug strategy. London: HM Government, 2008.

11. HM Government. Saving lives. Reducing harm. Protecting the public. An action plan for tackling violence 2008-11. London: HM Government, 2008.

12. Department of Health. Better prevention, better services, better sexual health - the national strategy for sexual health and HIV. London: Department of Health, 2001. tance with data input and all the young holidaymakers who voluntarily participated in this research. We would also like to thank the two anonymous reviewers who helped improve an earlier draft of this paper.

\section{Funding}

European Commission Directorate-General for Justice, Freedom and Security: Daphne 2006-1 (JLS/2006/Dap-1/069/ Y30-CE-0117583/00-55).

\section{Conflicts of interest: None declared.}

13. Bellis MA, Hughes $K$, Bennett $A$ et al. The role of an international nightlife resort in proliferation of recreational drugs. Addiction 2003; 98:1713-21.

14. Bellis MA, Hughes $K$, Thomson R and Bennett A. Sexual behaviour of young people in international tourist resorts. Sexually Transmitted Infections 2004; 80:43-47.

15. Josiam BM, Hobson JSP, Dietrich UC and Smeaton G. An analysis of the sexual, alcohol and drug related behavioural patterns of students on spring break. Tourism Management 1998; 19:01513

16. Maticka-Tyndale E, Herold ES and Oppermann M. Casual sex among Australian schoolies. Journal of Sex Research 2003; 40:158-169.

17. Hesse $M$, Tutenges $S$, Schliewe $S$ and Reinholdt T. Party package travel: alcohol use and related problems in a holiday resort: a mixed methods study. BMC Public Health 2008; 8:351.

18. Eiser JR and Ford N. Sexual relationships on holiday: a case of situational disinhibition? Journal of Social and Personal Relationships 1996; 12:323-339.

19. Lora-Tomayo C, Tena T and Rodríguez A et al. The designer drug situation in Ibiza. Forensic Science International 2004; 140:195206.

20. Henderson JC. Managing tourism crises. Burlington, MA: Butterworth-Heinemann, 2007.

21. BBC News. Youth moves on as Faliraki fades. 10th May 2004 Available at: http://news.bbc.co.uk/1/hi/uk/3700153.stm. Accessed 11th November 2008.

22. Forsyth A, Moyes $A$ and Young $H$. Increased ciprofloxacin resistance in gonococci isolated in Scotland. The Lancet 2000; 356:1984-1985.

23. Calafat $A$ and Juan $M$. Health and safety problems in recreational nightlife in the Island of Mallorca. International Journal of Drug Policy 2004; 15:157-162.

24. Dobruszkes F. An analysis of European low-cost airlines and their networks. Journal of Transport Geography 2006; 14:249-64.

25. National Statistics. Travel trends 2006: data and commentary from the international passenger survey. London: National Statistics, 2008.

26. Govern de les Illes de Balears. Dades informatives 2007: El turisme a les Illes Balears. Palma de Majorca: Govern de les Illes de Balears, 2008. 
27. Bellis MA, Hughes K, Dillon P, Copeland J and Gates P. Effects of backpacking holidays in Australia on alcohol, tobacco and drug use of UK residents. BMC Public Health 2007; 7:1.

28. Hughes $K$, Bellis MA, Calafat, $A$ et al. Predictors of violence in young tourists: a comparative study of British, German and Spanish holidaymakers. European Journal of Public Health. 2008;1-6.

29. Bellis MA, Hughes $K$, Calafat $A$, Juan $M$ and Schnitzer $S$. Relative contributions of holiday location and nationality to changes in recreational drug taking behaviour: a natural experiment in the Balearic Islands. European Addiction Research. En prensa.

30. Calafat A, Stocco P, Mendez F et al. Characteristics and social representation of ecstasy in Europe. Palma de Mallorca: IREFREA, 1998.

31. McCambridge J, Mitcheson $L$, Winstock $A$ et al. Five-year trends in patterns of drug use among people who use stimulants in dance contexts in the United Kingdom. Addiction 2005; 100:1140-1149.

32. Calafat A, Gomez CS, Juan M et al. Vida recreativa nocturna de los jóvenes espanoles como factor de riesgo frente a otros más tradicionales [Night life recreation of young Spaniards as a risk factor compared to other more traditional]. Adicciones 2007: 19;125-132.

33. Lucas B. La droga rompe la magia de Ibiza. El País. 17de octubre de 2005.

34. North West Public Health Observatory. Local Alcohol Profiles for England. Available at: http://www.nwph.net/alcohol/lape/. Accessed $12^{\text {th }}$ November 2008.

35. Office for National Statistics. Alcohol-related deaths in the United Kingdom. Available at: http://www.statistics.gov.uk/STATBASE/ Product.asp?vInk=14496. Accessed 12 ${ }^{\text {th }}$ November 2008.

36. Jones L, Bellis MA, Dedman D et al. Alcohol-attributable fractions for England: alcohol-attributable mortality and hospital admissions. Liverpool: Centre for Public Health, Liverpool John Moores University, 2008.

37. Cherpitel CJ. Alcohol and injuries: a review of international emergency room studies since 1995. Drug and Alcohol Review 2007; 26:201-214.

38. Lamminpää A. Alcohol intoxication in childhood and adolescence. Alcohol \& Alcoholism. 1995; 30: 5-12.

39. Simpson T, Murphy N and Peck DF. Saliva alcohol concentrations in accident and emergency attendances. Emergency Medicine Journal 2001:18; 250-254.
40. Engineer R, Phillips A, Thompson J et al. Drunk and disorderly: a qualitative study of binge drinking among 18- to 24-year-olds. London: Home Office, 2003.

41. Farke W, Anderson P. El consumo concentrado de alcohol en Europa [Binge drinking in Europe]. Adicciones 2007:19; 333340.

42. Hendrickson JC, Gerstein DR. Criminal involvement among young male ecstasy users. Substance Use and Misuse 2005:40; 155775.

43. Sumnall HR, Cole JC, Jerome L. The varieties of ecstatic experience: an exploration of the subjective experiences of ecstasy. Journal of Psychopharmacology 2006; 20: 670-682.

44 Livingstone M. A longitudinal analysis of alcohol outlet density and assault. Alcoholism: Clinical and Experimental Research 2008; 32:1074-1079

45. Graham K, Osgood DW, Wells S et al. To what extent is intoxication associated with aggression in bars? Journal of Studies on Alcohol 2006; 67:382-390.

46. Graham K, Bernards S, Osgood DW et al. Bad nights or bad bars? Multi level- analysis of environmental predictors of aggression in late night large-capacity bars and clubs. Addiction 2006; 101:1569-1580.

47. Hughes K, Bellis MA. Use of environmental harm to tackle alcoholrelated harm in nightlife environments: the UK experience. Lisbon: European Monitoring Centre of Drugs and Drug Addiction, 2007.

48. Are Brits abroad behaving badly? The Jeremy Kyle Show. ITV. aired $3^{\text {rd }}$ November 2008. Available at: http://www.itv.com/Lifestyle/ jeremykyle/default.html. Accessed 11th November 2008.

49. Cook PA and Bellis MA. Knowing the risk: relationships between risk behaviour and health knowledge. Public Health 2001; 115:54-61.

50. Johnson AM. Mercer $\mathrm{CH}$ and Erens B et al. Sexual behaviour in Britain: partnerships, practices, and HIV risk behaviours. The Lancet 2001; 358:1835-1842.

51. Cassell JA, Mercer $\mathrm{CH}$, Imrie J, et al. Who uses condoms with whom? Evidence from national probability sample surveys. Sexually Transmitted Infections 2006; 82:467-473.

52. Wellings K, Macdowall W, Catchpole M et al. Seasonal variations in sexual activity and their implications for sexual health promotion. Journal of the Royal Society of Medicine 1999; 92: 60-64. 
